\section{Buchbesprechung "Leben ohne Tod? Forscher besiegen das Altern"}

Eine Buchkritik wäre keine Buchkritik, wenn sie am Buch keinerlei Kritik üben würde. Norbert Welsch gibt einen exzellenten Abriss der aktuellen biogerontologischen Forschung, doch Titel und Untertitel sind höchst unglücklich. Nur wenige Wissenschaftler haben es sich zum Ziel gesetzt, den Tod abzuschaffen. Gesünder älter zu werden ist ja auch schon nicht schlecht.

„Forscher besiegen das Altern“ ist derzeit auch eine übertriebene Aussage. Der biologische Alterungsprozess wird zunehmend auf einer molekularen Ebene verstanden, das ist richtig. Aufbauend auf diesem Verständnis zeichnen sich auch erste hoffnungsvolle Strategien ab, das Altern zu beeinflussen. $\mathrm{Zu}$ behaupten, damit sei das Altern bereits besiegt, wäre aber in etwa so, als hätte jemand vor 50 Jahren angesichts der ersten Erkenntnisse über die Tumorbiologie behauptet, der Krebs sei bereits besiegt.

Die Kritik an dem reißerischen Titel ist nicht zuletzt auch deshalb wichtig, weil das Buch selbst einen ganz anderen Ton anschlägt. Welsch stellt wissenschaftlich durchaus anspruchsvoll, sehr aktuell und kritisch die Fortschritte der Altersforschung vor. So sind etwa die viel zitierten "freien Radikale“ und der mit ihnen verbundene „oxidative Stress“ offensichtlich gar nicht so negativ, wie lange dargestellt. Freie Radikale spielen vielmehr eine wichtige Rolle in unserem Immunsystem. Ihnen kommt auch eine Schlüsselfunktion in der Krebsabwehr zu. Ein wahlloses Herunterregeln der freien Radikale ist daher alles andere als sinnvoll.

Gleichzeitig zeigt Welsch auch neue Ansätze auf. So werden etwa Signalwege - oder besser gesagt, die Störung von Signalwegen - für das Verständnis von Alterungsprozessen immer wichtiger. Beispielhaft sei hier die gestörte Signaltransduktion zwischen Zellkern und Mitochondrien genannt, die sich immer mehr als wesentlich für die zelluläre Seneszens herausstellt. All dies wird von Welsch detailliert auf einer molekularbiologischen Grundlage dargestellt.

Gewünscht hätte ich mir noch eine Auseinandersetzung mit der Arbeit von
"Leben ohne Tod?

Forscher besiegen das Altern"

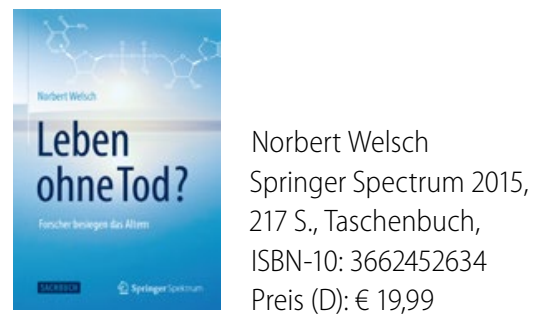

Aubrey de Grey. Der exzentrische britische Biogerontologe mit seinem SENS(Strategies of Engeneered Negligible Senescense)-Programm gilt inzwischen als Wortführer der „Radical Life Extension“. Sein Buch „Ending Aging“ ist so etwas wie die Blaupause für ein Leben ohne Tod. Auch wenn man seinem Konzept kritisch gegenübersteht, sollte es in einer Veröffentlichung, welche die Abschaffung des Alterns in Aussicht stellt, zumindest Erwähnung finden. Diesbezüglich hoffen wir dann einmal auf die zweite Auflage. Lesenswert ist aber auch schon die erste.

Prof. Dr. med. Bernd Kleine-Gunk, Fürth

\title{
Buchbesprechung „Dermatological Diseases and Cumulative Life Course Impairment"
}

Denis Linder, langjähriger Präsident der Europäischen Vereinigung für Dermatologie und Psychiatrie (ESDaP), hat zusammen mit Alexa Kimball ein Buch herausgegeben, das das Thema Lebensqualität und belastende Lebensereignisse und deren Auswirkungen auf chronische Hauterkrankungen beschreibt.

Im ersten Teil des Buches werden Modelle zur Lebensplanung und der grundsätzlichen Bewertung von Lebensereignissen dargestellt. Die Lebensverläufe lassen sich einerseits durch kumulative Lebensereignisse, aber auch durch eine sogenannte „Allostasis“ beschreiben. Die Allostasis ist gekennzeichnet durch die psychologische Vorstellung, dass zunächst Stress einen positiven Einfluss auf die Lebensqualität ausübt, allerdings wenn ein individuelles Maß überschritten ist, sich negative Auswirkungen auf unterschied- liche Organe zeigen können. Die Interaktion mit chronischen Erkrankungen ist komplex und lässt sich durch gesellschaftliche, soziale, politische und ökonomische Einflussfaktoren charakterisieren.

Im zweiten Teil des Buches werden die kumulativen Lebensereignisse im Verhältnis zur Lebensqualität dargestellt. Zur Beurteilung können hier vorgestellte Fragebögen Anwendung finden.

Schließlich sind im dritten und letzten Teil an verschiedenen häufigen chronischen Erkrankungen der Haut Zusammenhänge zu kumulativen Lebensereignissen dargestellt und werden näher beschrieben. Darüber hinaus werden abschließend gesellschaftliche und kulturelle Einflüsse auf die Lebensqualität diskutiert.

Zusammenfassend lässt sich sagen, dass sich das Buch mit moderner Lebens-
"Dermatological Diseases and Cumulative Life Course Impairment"

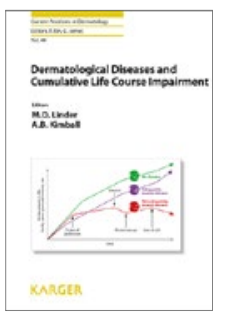

Denis Linder, Alexa

Kimball

Karger 2013, 162 S., ISBN: 978-3-318-02403-6 Preis (D): $€ 196,90$

qualitätsforschung bei chronischen Hauterkrankungen beschäftigt, ein Thema, dass sowohl aus psychodermatologischer als auch aus gesellschaftlich-ökonomischer Sicht als hochaktuell und bedeutungsvoll darstellt. All denjenigen wissenschaftlich tätigen Kollegen, die sich mit der Lebensqualität in der Dermatologie beschäftigen, sei dieses Buch besonders ans Herz gelegt.

Prof. Dr. Klaus-Michael Taube, Halle (Saale) 